\title{
Joint Determination of Power and Decoding Order for Successive Inter- and Intra-Cell Interference Cancellation
}

\author{
Hye Won Chung, Sang-Woon Jeon, Do-Hyung Park, and Sae-Young Chung \\ School of EECS, Korea Advanced Institute of Science and Technology, Daejeon, Korea \\ E-mail: \{hwisland, swjeon, dohyungpack\}@kaist.ac.kr, sychung@ee.kaist.ac.kr
}

\begin{abstract}
The performance of multiple access systems mainly depends on the management of interference arising from both intra-cell and inter-cell transmission. Successive interference cancellation (SIC) is a promising approach to manage interference in both the practical and information theoretic senses. Because the messages of the cell boundary users are likely to be decoded not only at the serving base station but also at an adjacent base station, it is possible to subtract inter-cell interference of the cell boundary users before SIC of the intra-cell users. This is the motivation of our work. In this paper, we first decode the messages of the adjacent cell boundary users and subtract them, and then perform SIC within the serving users. It shows better performance compared to the conventional schemes with the SIC within the serving users only.
\end{abstract}

\section{INTRODUCTION}

In the cellular systems, the transmit power control has been an essential technique, i.e., code division multiple access (CDMA) systems, since interference from other users degrades the system performance. In order to support data services such as a file or image download, current cellular systems allocate more resources for the downlink traffic than for the uplink traffic. Due to the increasing downlink traffic, most of the previous works of the power control and rate allocation has been concentrated on the downlink environments [1], [2]. More recently, however, the demand of the uplink traffic also increases proportional to the downlink traffic. Due to the dynamic downlink resource allocation, more channel feedbacks or control messages are needed. Furthermore, some applications such as a file or image upload require large amount of uplink resources. Based on this consideration, some works [3], [4], [5] related to the uplink power control and rate allocation have been performed.

In direct sequence CDMA systems, multiple-access interference (MAI) causes performance loss due to the nonorthogonality of spreading codes. Successive interference cancellation (SIC) is one of the promising methods as a practical approach toward multiuser detection (MUD). The iterative power controls for SIC are proposed in [6], [7] and the effect of a channel estimation error is considered in [8]. Power controls based on the multirate direct sequence CDMA systems are considered under non-fading and fading channels in [9].

It is known that, for a given transmit power constraint, SIC combined with the minimum mean square error (MMSE) detection can achieve the sum-rate capacity [10]. In this

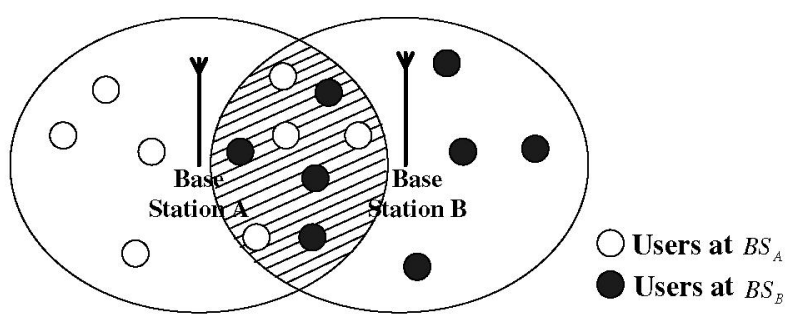

Fig. 1. Two adjacent cells with overlap region.

paper, we consider the scheme which performs SIC not only within the intra-cell users but also within the intercell users who are at cell boundaries. By employing partial SIC of the inter-cell users, it is possible to improve system performances.

\section{System ModeL}

We consider an uplink multi-cell cellular system in which there exists intra-cell and inter-cell interference. In cellular systems, coverages of each cell are overlapped with the adjacent cells since it is impossible to make a beam to the perfect hexagonal shape. The messages of cell-boundary users, which are located in the shaded region in Fig. 1, are likely to be decoded from both base stations since the cellboundary users are usually supported with a low rate, and their channel gain to the serving base station (BS) is similar to the channel gain to the adjacent BS.

In this paper, we simplify the multi-cell system to an one dimensional network and consider only one adjacent BS as shown in Fig. 2. Two BSs are separated by distance $2 R$ and the coverage of each cell is up to $(1+\alpha) R$, where $\alpha$ denotes the overlap factor. $B S_{A}$ denotes the serving BS for users served by it and $B S_{B}$ denotes the adjacent $B S$ whose serving users cause inter-cell interference to $B S_{A}$. There are $N$ number of users in $B S_{A}$ and the users are uniformly distributed in $(1+\alpha) R$ region. It is assumed that, $B S_{B}$, has the mirror users corresponding to the serving users in $B S_{A}$, which means that the mirror user locates with $(2 R-r)$ distance apart from the origin when the serving user is located at $r$. This assumption is reasonable if there exists many users in each cell and only a small fraction of interference caused by the adjacent cell's users is canceled.

Let $r_{k}$ denotes the distance from the origin to the $k^{\text {th }}$ 


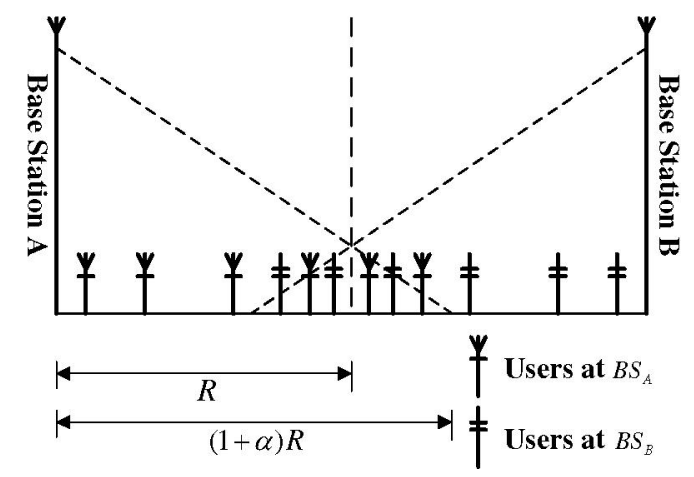

Fig. 2. One-dimensional distribution of users between two base stations.

serving user. The channel gain of the $k^{\text {th }}$ serving user is given by

$$
g_{k}=\frac{1}{r_{k}^{\beta}}
$$

where $\beta$ is the path loss exponent. The channel gain of the $k^{\text {th }}$ mirror user is given by

$$
g_{k}^{o}=\frac{1}{\left(2 R-r_{k}\right)^{\beta}}
$$

When SIC is employed for inter-cell and intra-cell interference cancellation, the reverse link signal-to-interferenceplus-noise ratio (SINR) of the $\pi(k)^{\text {th }}$ serving user can be represented as

$$
\begin{aligned}
& S I N R_{\pi(k)}= \\
& \quad \frac{g_{\pi(k)} P_{\pi(k)}}{N_{0}+I_{e}+\sum_{j=k+1}^{N} g_{\pi(j)} P_{\pi(j)}}, \quad \forall k \in[1, N]
\end{aligned}
$$

where $\Pi=\{\pi(1), \cdots, \pi(N)\}$ is the ordered set that indicates the decoding order of the serving users. $P_{\pi(k)}$ is the transmit power of the $\pi(k)^{\text {th }}$ serving user and $N_{0}$ is the noise variance. If we cancel inter-cell interference up to $M$ mirror users, the inter-cell interference $I_{e}$ is given by $\sum_{j=1}^{N} g_{j}^{o} P_{j}-$ $\sum_{j=1}^{M} g_{\pi^{\circ}(j)}^{o} P_{\pi^{\circ}(j)}$, where $\Pi^{o}=\left\{\pi^{o}(1), \cdots, \pi^{o}(M)\right\}$ is the ordered set that indicates the decoding order of the mirror users. Since the channel gain and interferer distribution between the serving user and $B S_{A}$ is symmetric with that between the corresponding mirror user and $B S_{B}$, the transmit power $P_{i}$ of the $i^{\text {th }}$ serving user is equal to $P_{i}^{o}$ for all $i \in[1, N]$, where $P_{i}^{o}$ is the transmit power of the $i^{\text {th }}$ mirror user. It is assumed that the message of the $\pi(k)^{\text {th }}$ user can be decoded if $S I N R_{\pi(k)}>\gamma$, where $\gamma$ denotes the target SINR value.

In order to decode $M$ mirror users, the additional constraints such as $S I N R_{\pi^{\circ}(k)}^{o}>\gamma$ should be satisfied for all $k \in[1, M]$. The SINR of the $\pi^{o}(k)^{\text {th }}$ mirror user is given by

$$
\begin{aligned}
& S I N R_{\pi^{\circ}(k)}^{o}= \\
& \quad \frac{g_{\pi^{o}(k)}^{o} P_{\pi^{\circ}(k)}}{N_{0}+\sum_{j=1}^{N} g_{j} P_{j}+\sum_{j=k+1}^{M} g_{\pi^{\circ}(j)}^{o} P_{\pi^{\circ}(j)}+I_{e}}, \quad \forall k \in[1, M]
\end{aligned}
$$

In this paper, we consider the following problem of finding the transmission power and the decoding order for intercell and intra-cell interference cancellation that minimizes the total power consumption:

$$
f\left(\mathbf{P}, \Pi, \Pi^{\circ}\right)=\min _{\mathbf{P}, \Pi, \Pi^{\circ}, M} \sum_{k=1}^{N} P_{\pi(k)}
$$

subject to

$$
\begin{aligned}
S I N R_{\pi(k)} & \geq \gamma, \quad \forall k \in[1, N] \\
S I N R_{\pi(k)}^{o} & \geq \gamma, \quad \forall k \in[1, M] \\
P_{\pi(k)} & \leq P_{\max , \pi(k)}, \quad \forall k \in[1, N]
\end{aligned}
$$

where $\mathbf{P}=\left[P_{\pi(1)}, \cdots, P_{\pi(N)}\right]^{T}$ is the transmit power vector, $\mathbf{P}_{\max }=\left[P_{\max , \pi(1)}, \cdots, P_{\max , \pi(N)}\right]^{T}$ is the maximum transmit power vector, and $M$ is equal to the cardinality of $\Pi^{\circ}$.

\section{POWER ALLOCATION FOR INTRA-AND INTER-CELL CANCELLATION}

\section{A. Decoding Order and Corresponding Power Assignment}

First, let us assume that we do not employ inter-cell interference cancellation. Then, the inter-cell interference $I_{e}$ is given by $\sum_{j=1}^{N} g_{j}^{o} P_{j}$. From (3) and (6), the transmit power of each user should satisfy the following equation:

$$
\mathbf{A G P} \geq \mathbf{B}
$$

where $\mathbf{G}$ is a diagonal matrix with the $i^{\text {th }}$ diagonal element of $g_{\pi(i)}$ and $\mathbf{B}=\left[\gamma N_{0}, \ldots, \gamma N_{0}\right]^{T}$. $\mathbf{A}$ is given by (12).

By assuming perfect power control, i.e., $S I N R_{\pi(k)}=\gamma$ for all $k \in[1, N]$, we can find the minimum transmission power vector which satisfies (9) with equality. Therefore, the transmit power of the $\pi(k)^{\text {th }}$ user is obtained as

$$
P_{\pi(k)}=\frac{\frac{1}{g_{\pi(k)}}\left(\frac{1}{1+\gamma}\right)^{k-1} \times}{(1+\gamma)^{1-N}-\gamma \sum_{j=1}^{N}\left(\frac{1}{1+\gamma}\right)^{j-1} \frac{g_{\pi(j)}^{o}}{g_{\pi(j)}}}
$$

Lemma 1: In order to minimize the total power consumption by using intra-cell interference cancellation, the serving users should be decoded in the order of their channel gain.

Proof: Let's consider the serving user $u_{1}$ and $u_{2}$ whose channel gains satisfy the relationship, $g_{u_{1}}>g_{u_{2}}$. Suppose the $u_{1}$ 's message is decoded and canceled at the $k^{\text {th }}$ order and next the $u_{2}$ 's message is decoded. The assigned power for each user is given by

$$
\begin{aligned}
& P_{u_{1}}=\quad \frac{1}{g_{u_{1}}}\left(\frac{1}{1+\gamma}\right)^{k-1} \times \\
& \frac{\gamma N_{0}}{c-\gamma\left(\frac{1}{1+\gamma}\right)^{k-1}\left(\frac{g_{u_{1}}^{o}}{g_{u_{1}}}+\left(\frac{1}{1+\gamma}\right) \frac{g_{u_{2}}^{o}}{g_{u_{2}}}\right)} \\
& P_{u_{2}}=\quad \frac{1}{g_{u_{2}}}\left(\frac{1}{1+\gamma}\right)^{k} \times \\
& \frac{\gamma N_{0}}{c-\gamma\left(\frac{1}{1+\gamma}\right)^{k-1}\left(\frac{g_{u_{1}}^{o}}{g u_{1}}+\left(\frac{1}{1+\gamma}\right) \frac{g_{u_{2}}^{o}}{g u_{2}}\right)}
\end{aligned}
$$




$$
\begin{aligned}
& \mathbf{A}=\left(\begin{array}{ccccc}
1-\gamma \frac{g_{\pi(1)}^{\circ}}{g_{\pi(1)}} & -\gamma\left(1+\frac{g_{\pi(2)}^{\circ}}{g_{\pi(2)}}\right) & \ldots & -\gamma\left(1+\frac{g_{\pi(N-1)}^{\circ}}{g_{\pi(N-1)}}\right) & -\gamma\left(1+\frac{g_{\pi(N)}^{o}}{g_{\pi(N)}}\right) \\
-\gamma \frac{g_{\pi(1)}^{o}}{g_{\pi(1)}} & 1-\gamma \frac{g_{\pi(2)}^{\circ}}{g_{\pi(2)}} & \vdots & \vdots & \vdots \\
\vdots & -\gamma \frac{g_{\pi(2)}^{\circ}}{g_{\pi(2)}} & \ddots & -\gamma\left(1+\frac{g_{\pi(N-1)}^{\circ}}{g_{\pi(N-1)}}\right) & \vdots \\
\vdots & \vdots & \ldots & 1-\gamma \frac{g_{\pi(N-1)}^{\circ}}{g_{\pi(N-1)}} & -\gamma\left(1+\frac{g_{\pi(N)}^{o}}{g_{\pi(N)}}\right) \\
-\gamma \frac{g_{\pi(1)}^{o}}{g_{\pi(1)}} & -\gamma \frac{g_{\pi(2)}^{o}}{g_{\pi(2)}} & \ldots & -\gamma \frac{g_{\pi(N-1)}^{\circ}}{g_{\pi(N-1)}} & 1-\gamma \frac{g_{\pi(N)}^{\circ}}{g_{\pi(N)}}
\end{array}\right) \\
& \sum_{j=1}^{N} P_{j}-\sum_{j=1}^{N} P_{j}^{\prime}=\left(P_{u 1}+P_{u 2}\right)-\left(P_{u 1}^{\prime}+P_{u 2}^{\prime}\right) \\
& =\left(\frac{1}{1+\gamma}\right)^{k-1}\left[\frac{\gamma N_{0}}{c-\gamma\left(\frac{1}{1+\gamma}\right)^{k-1}\left\{\frac{g_{u 1}^{\circ}}{g_{u 1}}+\left(\frac{1}{1+\gamma}\right) \frac{g_{u 2}^{o}}{g_{u 2}}\right\}}\left\{\frac{1}{g_{u 1}}+\left(\frac{1}{1+\gamma}\right) \frac{1}{g_{u 2}}\right\}\right. \\
& \left.-\frac{\gamma N_{0}}{c-\gamma\left(\frac{1}{1+\gamma}\right)^{k-1}\left\{\frac{g_{u 2}^{o}}{g_{u 2}}+\left(\frac{1}{1+\gamma}\right) \frac{g_{u 1}^{\circ}}{g_{u 1}}\right\}}\left\{\frac{1}{g_{u 2}}+\left(\frac{1}{1+\gamma}\right) \frac{1}{g_{u 1}}\right\}\right]
\end{aligned}
$$

where $c$ is a constant that is not changed according to the decoding order of user $u_{1}$ and $u_{2}$. If the message of the serving user $u_{2}$ is canceled before decoding the serving user $u_{1}$ 's message, the assigned power for each user, which are denoted as $P_{u_{1}}^{\prime}$ and $P_{u_{2}}^{\prime}$ respectively, are obtained by changing the variable $u_{1}$ to $u_{2}$ and $u_{2}$ to $u_{1}$ in (11). The difference of the sum power between two strategies is given by (13).

From (1) and (2), we can derive that if $g_{u_{1}}>g_{u_{2}}$, then $g_{u_{1}}^{o}<g_{u_{2}}^{o}$. Therefore, (13) has a negative value. This means that the sum power, which is calculated by assuming that the user $u_{1}$ 's message is canceled before user $u_{2}$ 's message, is smaller than that of being calculated by assuming the reverse decoding order. In conclusion, in order to minimize the total power consumption the serving users should be decoded in the order of their channel gain.

If we perform SIC for intra-cell interference in the order of their channel gain, the assigned power for each user is given by (10) with $\Pi$ such that $g_{\pi(1)}>g_{\pi(2)}>\cdots>g_{\pi(N)}$.

Next, let's consider inter-cell interference cancellation. If we assume that $M$ mirror users are canceled, $g_{\pi(j)}^{o}=0$ for $\pi(j) \in \Pi^{\circ}$ in (10). Therefore, the required power for each user decreases as $M$ increases. However, the additional constraints, which is given by (4) and (7), should be satisfied to employ inter-cell interference cancellation. Even though the decoding order of the mirror users does not affect the SINR values of the serving users, the SINR values of the mirror users in (4) depend on $\Pi^{\circ}$.

Let's consider two mirror users $v_{1}$ and $v_{2}$ whose channel gains satisfy the relationship, $g_{v_{1}}^{\circ}>g_{v_{2}}^{o}$. Suppose that $P_{\pi(k)}$ is the transmit power that satisfies $S I N R_{\pi(k)}=\gamma$ for all $k \in[1, N]$ with inter-cell interference cancellation of $v_{1}$ and $v_{2}$ mirror users. Since $g_{v_{1}}^{o}>g_{v_{2}}^{o}$, it is reasonable to cancel the message of $v_{1}$ mirror user first, and then decode that of the $v_{2}$ mirror user to make $I_{v_{1}}^{o}>I_{v_{2}}^{o}$ under the given $\left\{P_{\pi(k)}, k \in[1, N]\right\} . I_{v_{1}}^{o}$ and $I_{v_{2}}^{o}$ are the total interference when $B S_{A}$ decodes the $v_{1}$ and $v_{2}$ mirror users' message, respectively. With this ordering, the SINR values for mirror users are balanced, so that it is more likely to satisfy the SINR constraints in (7). Therefore, $\Pi^{\circ}$ should be ordered to meet the condition, $g_{\pi^{\circ}(1)}^{o}>g_{\pi^{\circ}(2)}^{o}>\cdots>g_{\pi^{\circ}(M)}^{o}$.

In conclusion, the ordered set $\Pi$ and $\Pi^{\circ}$ should satisfy $g_{\pi(1)}>g_{\pi(2)}>\cdots>g_{\pi(N)}$ and $g_{\pi^{\circ}(1)}^{o}>g_{\pi^{\circ}(2)}^{o}>\cdots>$ $g_{\pi^{\circ}(M)}^{o}$, respectively. Since the serving users and its mirror users are located symmetrically, $\pi^{o}(k)$ is equal to $(N+1-$ $\pi(k))$ for all $k \in[1, M]$. Applying this relationship to (10), the transmit power of $\pi(k)^{\text {th }}$ user can be represented as

$$
P_{\pi(k)}=\frac{\frac{1}{g_{\pi(k)}}\left(\frac{1}{1+\gamma}\right)^{k-1} \times}{(1+\gamma)^{1-N}-\gamma \sum_{j=1}^{N-M}\left(\frac{1}{1+\gamma}\right)^{j-1} \frac{g_{\pi(j)}^{o}}{g_{\pi(j)}}}
$$

The SINR condition for the $M$ mirror users can also be simplified by applying $\pi^{o}(k)=N+1-\pi(k)$ to (4) and (7),

$$
\begin{aligned}
& \operatorname{SINR}_{\pi(k)}^{o}= \\
& \frac{g_{\pi(k)}^{o} P_{\pi(k)}}{N_{0}+\sum_{j=1}^{N} g_{j} P_{j}+\sum_{j=1}^{k-1} g_{\pi(j)}^{\circ} P_{\pi(j)}}, \\
& \forall k \in[N+1-M, N]
\end{aligned}
$$

B. Efficient Power Allocation for Saving Total Power Consumption

In this section, we propose a suboptimal algorithm to find the maximum number of mirror users which are canceled before intra-cell interference. The corresponding power allocation is also be determined. The ordered set $\Pi$, which is suggested in the previous section, is used in the following Lemma 2. Since $g_{\pi(1)}>g_{\pi(2)}>\cdots>g_{\pi(N)}$ in $\Pi$, $g_{\pi(N)}^{o}>g_{\pi(N-1)}^{o}>\cdots>g_{\pi(1)}^{o}$. Therefore, we will cancel inter-cell users in the descending order from $\pi(N)$ to $\pi(N+1-M)$, where $M$ is the number of inter-cell users which are canceled.

There exists an upper bound for $M$, denoted by $M_{\text {upper }}$. $M_{\text {upper }}$ is the number of the mirror users whose channel 


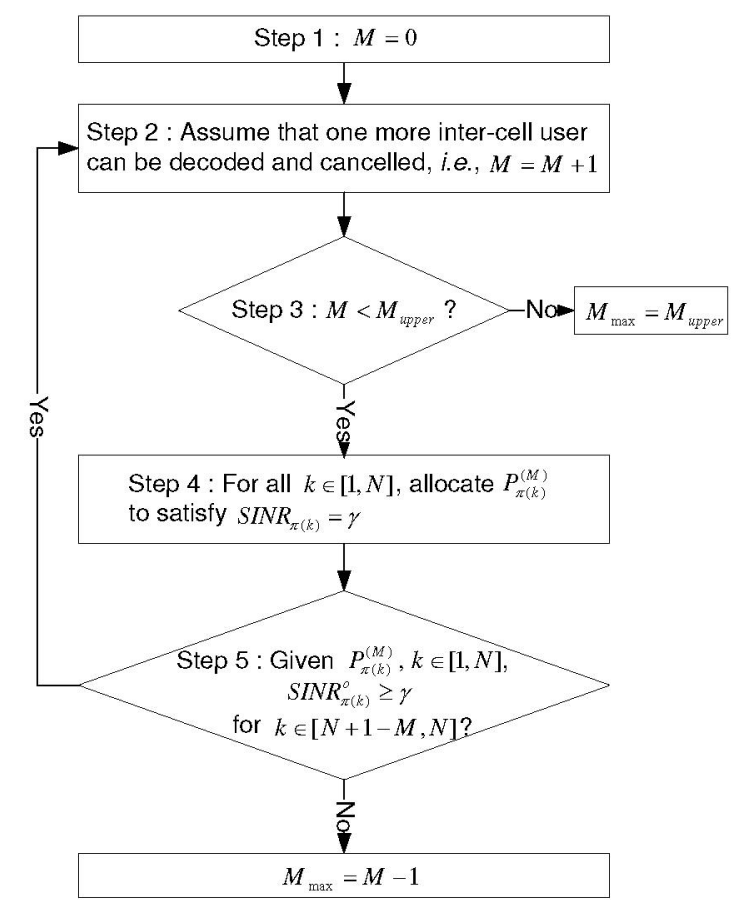

Fig. 3. Flow chart for determining the maximum value of $M$.

gain meets the condition $g_{\pi(k)}^{o}>g_{\pi(k)}$. For all $k \in[N+$ $1-M, N]$, in order to be $S I N R_{\pi(k)}^{o} \geq S I N R_{\pi(k)}=\gamma$, it is necessary to satisfy $g_{\pi(k)}^{o}>g_{\pi(k)}$.

Lemma 2: If SINR in (15) is larger than $\gamma$ assuming $M_{0}+1$ mirror users were canceled, i.e., $M=M_{0}+1$, with $\left\{P_{\pi(k)}^{\left(M_{0}+1\right)}, k \in[1, N]\right\}$, then the target SINR is also satisfied for the case assuming $M_{0}$ mirror users were canceled, i.e., $M=M_{0}$, with $\left\{P_{\pi(k)}^{\left(M_{0}\right)}, k \in[1, N]\right\} . P_{\pi(k)}^{(M)}$ denotes the assigned power of the $\pi(k)^{\text {th }}$ serving user assuming $M$ mirror users were canceled.

Proof: From (14), $P_{\pi(k)}^{\left(M_{0}+1\right)}=\rho P_{\pi(k)}^{\left(M_{0}\right)}$ for all $k \in$ $[1, N]$, where $\rho(0<\rho<1)$ does not depend on $k$. By substituting $P_{\pi(k)}^{\left(M_{0}+1\right)}$ and $P_{\pi(k)}^{\left(M_{0}\right)}$ in (15), and using $P_{\pi(k)}^{\left(M_{0}+1\right)}=$ $\rho P_{\pi(k)}^{M_{0}}$, we can get $S I N R_{\pi(k)}^{o\left(M_{0}+1\right)}<S I N R_{\pi(k)}^{o\left(M_{0}\right)}$ for all $k \in\left[N+1-M_{0}, N\right] . S I N R_{\pi(k)}^{o(M)}$ is the SINR of the $\pi(k)^{\text {th }}$ mirror user, assuming $M$ mirror users were canceled. Therefore, if $S I N R_{\pi(k)}^{o\left(M_{0}+1\right)} \geq \gamma, \forall k \in\left[N+1-\left(M_{0}+\right.\right.$ 1), N], then $S I N R_{\pi(k)}^{o\left(M_{0}\right)} \geq \gamma, \forall k \in\left[N+1-M_{0}, N\right]$. In conclusion, if it is possible to employ SIC for $\left(M_{0}+1\right)$ mirror users, then it is also possible to employ SIC for $M_{0}$ mirror users.

Based on Lemma 2, a suboptimal algorithm to determine $M_{\max }$ is proposed in Fig. 3, where $M_{\max }$ denotes the number of the mirror users that can be decoded and subtracted at the serving BS.

It is assumed that the total number of users assigned to each BS is $N$. In Step 1, the initial value of $M$ is set to zero. In Step 2, it is assumed that one more mirror user can be decoded and subtracted. The value of $M$ is checked in
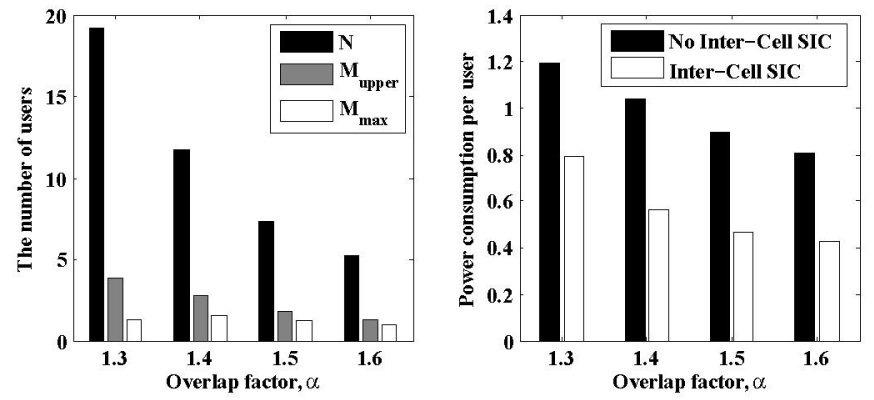

Fig. 4. The effect of overlap factor in the reduction ratio of transmit power per user.
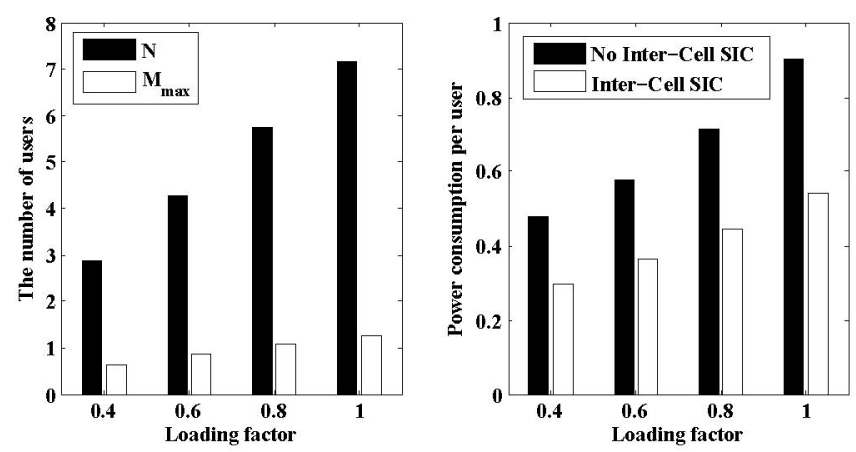

Fig. 5. The effect of loading factor in the reduction ratio of transmit power per user.

Step 3, whether it exceeds the upper bound for $M$, denoted by $M_{\text {upper }}$, or not. If $M<M_{\text {upper }}$, the simulation proceeds to Step 4, but if this condition is not satisfied, $M_{\max }$ equals

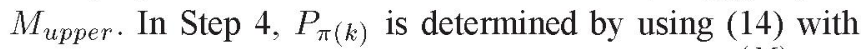
the assumed value of $M$. In Step 5, with given $\left\{P_{\pi(k)}^{(M)}, k \in\right.$ $[1, N]\}$, SINR for the mirror users are checked whether all these values satisfy the target $\gamma$, or not. If the condition is satisfied, simulation goes back to Step 2 to increase the value of $M$, and to check whether or not these $M$ mirror users can be canceled and decoded at the serving BS. If the condition in Step 5 is not satisfied, the simulation is finished and $M_{\max }$ is set as $(M-1)$.

\section{Simulation Results}

In this section, computer simulation results are given to verify the effectiveness of inter-cell interference cancellation in the reduction of transmit power per user. In order to verify the effectiveness of inter-cell SIC scheme, the following simulations show the reduction ratio of transmit power per user between the schemes with and without inter-cell SIC. Moreover, the reduction ratio of power is compared according to different overlap factors and loading factors.

The maximum number $N$ of users in each cell, the maximum number $M_{\text {max }}$ of inter-cell users which can be decoded and canceled, and the upper bound $M_{\text {upper }}$ for $M$ are compared with respect to different overlap factor $\alpha$ when only intra-cell SIC is employed, in the left graph of Fig. 4. 
As two adjacent cells are more overlapped, the maximum number of users, which meet the target SINR and power constraints, decreases. It is because the effect of intercell interference increases with overlap factor $\alpha$. However, the ratio of $M_{\max }$ to $N$ increases as two cells are more overlapped. Therefore, by employing inter-cell SIC scheme, the reduction ratio of power consumption per user increases with overlap factor $\alpha$ as shown in the right graph of Fig. 4 .

Next, with fixed overlap factor $\alpha=1.5$, the effect of loading factor on the number $N$ of users in each cell and the maximum number $M_{\max }$ of canceled inter-cell users are analyzed in the left graph of Fig. 5. $M_{\max }$ is slightly increased with the loading factor. The power saving ratio is almost same for the loading factors from 0.4 to 1 as shown in the right graph of Fig. 5.

\section{Conclusions}

A new inter- and intra-cell SIC scheme, which reduces total transmit power compared to the general intra-cell SIC scheme, is proposed. Power and decoding order for inter- and intra-cell users are jointly determined in order to minimize total power consumption. A suboptimal algorithm to find the maximum number of inter-cell users, which can be decoded and canceled at the serving base station, is suggested. By using this algorithm, we show that the proposed SIC scheme improves system performance.

\section{ACKNOWLEDGEMENT}

This work was supported by the center for Broadband OFDM Mobile Access (BrOMA) at POSTECH through the ITRC program of the Korean MIC, supervised by IITA. (IITA-2006-C1090-0603-0037) and by the Basic Research Program of the Korea Science \& Engineering Foundation (R01-2006-000-11112-0).

\section{REFERENCES}

[1] P. Bender et al, "CDMA/HDR A Bandwidth Efficient High Speed Wireless Data Service for Nomadic Users" IEEE Communications Magazine, 2000.

[2] D. M. Andrews, K. Kumaran, K. Ramanan, A. Stolyar, R. Vijayakumar, and P. Whiting, "CDMA Data QoS Scheduling on the Forward Link with Variable Channel Conditions," Bell Labs Technical Memo No.10009626-000404-05TM, 2000.

[3] F. Berggren and S. L. Kim, "Energy-Efficient Control of Rate and Power in DS-CDMA Systems," IEEE Transactions on Wireless Communication, vol. 3, NO.3, pp. 725-733, May 2004.

[4] K. Kumaran and L. Qian, "Uplink Scheduling in CDMA Packet-Data Systems," IEEE Infocom, vol. 1, pp. 292-300, Mar. 2004.

[5] H. Nie, P. T. Mathiopoulos and G. K. Karagiannidis, "Reverse Link Capacity Analysis of Cellular CDMA Systems with Controlled Power Disparities and Successive Interference Cancellation," IEEE Transactions on Wireless Communication, vol. 5, NO.9, pp. 292-300, pp. 2447-2457, Sep. 2006.

[6] F. Berggren and S. Ben Slimane, "Power Allocation for a Simple Successive Interference Cancellation Scheme in a MultiRate DS-CDMA System," IEEE Int. Conf. Communication, vol. 1, pp. 351-355, Apr. 2002.
[7] J. Andrews, A. Agrawal, T. Meng, and J. Cioffi, "A simple Iterative Power Control for Successive Interference Cancellation," IEEE Int. Symp. Spread Spectrum Techniques and Applications, vol. 3, pp. 761-765, 2002.

[8] A. Agrawal, J. G. Andrews, J. M. Cioffi and T. Meng, "Iterative Power Control for Imperfect Successive Interference Cancellation," IEEE Trans. Wirelss Communication, vol. 4, Issue 3, pp. 878-884, May 2005.

[9] H. Li, H. V. Poor, "Power Allocation, Decoding Order and Spectral Efficiency of Successive Interference Cancellation Based Multirate DS-CDMA Systems," IEEE Conf. Globecom, pp. 774-778, June 2004.

[10] S. Shamai and S. Verdu, "The impact of frequency-flat fading on the spectral efficiency of CDMA," IEEE Trans. Inform. Theory, vol 47, pp. 1302-1327, Mar. 2001. 